\title{
Single whispering-gallery mode lasing in polymer bottle microresonators via spatial pump engineering
}

\author{
Fuxing $\mathrm{Gu}^{1}$, Fuming $\mathrm{Xie}^{1}$, Xing Lin $^{2}$, Shuangyi Linghu' ${ }^{1}$, Wei Fang ${ }^{2}$, Heping Zeng ${ }^{1,3}$, Limin Tong ${ }^{2}$ \\ and Songlin Zhuang ${ }^{1}$
}

Single-mode lasing in whispering-gallery mode (WGM) microresonators is challenging to achieve. In bottle microresonators, the highly non-degenerated WGMs are spatially well-separated along the long-axis direction and provide mode-selection capability. In this work, by engineering the pump intensity to modify the spatial gain profiles of bottle microresonators, we demonstrate a simple and general approach to realizing single-mode WGM lasing in polymer bottle microresonators. The pump intensity is engineered into an interference distribution on the bottle microresonator surface. By tuning the spacing between axial positions of the interference pump patterns, the mode intensity profiles of single-bottle WGMs can be spatially overlapped with the interference stripes, intrinsically enabling single-mode lasing and selection. Attractive advantages of the system, including high sidemode suppression factors $>20 \mathrm{~dB}$, large spectral tunability $>8 \mathrm{~nm}$, low-lasing threshold and reversible control, are presented. Our demonstrated approach may have a variety of promising applications, ranging from tunable single-mode lasing and sensing to nonlinear optics.

Light: Science \& Applications (2017) 6, e17061; doi:10.1038/Isa.2017.61; published online 6 October 2017

Keywords: bottle microresonators; polymer; single-mode lasing; spatial pump engineering; whispering-gallery modes

\section{INTRODUCTION}

Owing to its high-beam quality and spectral purity, single-mode lasing is of fundamental importance for various scientific and technical applications. To achieve single-mode operation, some additional optical elements and strategies are required to suppress other competing modes in a lasing cavity. Owing to their high-quality factors and small-mode volumes, whispering-gallery mode (WGM) microresonators fabricated with different geometries (for example, microdisks, microspheres and microtoroids) and materials (for example, glass, semiconductor and polymer) have attracted increasing attention in various areas, including in lasing, sensing and optical communications $^{1-8}$. Generally, WGM lasers, which are generated through total internal reflection at the external cavity interface, are usually multimodal due to the lack of mode-selection strategies. To obtain single-mode WGM lasing, one possible strategy is to decrease the cavity dimensions and expand the free-space range (FSR) of the multimodes until only one resonant mode is left; however, this method will reduce the round-trip gain and increase the lasing threshold ${ }^{9}$. Other strategies, such as using two coupled cavities based on the Vernier effect ${ }^{10-13}$ and the parity-time symmetry effect ${ }^{14,15}$, have been reported; however, these methods usually require complicated and rigorous fabrication and precise manipulation.

Recently, prolate-shaped bottle microresonators, fabricated from optical glass fibers and capillaries, have attracted much attention and have been applied in interesting ways, such as for compact optical delay lines, cavity optomechanics, electromagnetically induced transparencylike phenomena and optical frequency comb generation ${ }^{16-23}$. Compared with other WGM resonator shapes, the unique advantage of bottle microresonators is that their prolate shapes support highly nondegenerated WGMs with spatially well-separated intensity along the long-axis direction, making it possible to select one desired mode as the dominant lasing mode according to their axial-mode numbers and thus suppress all other modes ${ }^{24}$. In recent years, the direct modification of the surfaces of passive glass bottle microresonators, such as adding liquid drops and inscribing microstructures on the resonator surfaces, to reduce the number of WGMs has been performed ${ }^{25,26}$. Nevertheless, it is difficult to incorporate gain materials into these types of glass microresonators for lasing. To our knowledge, single-mode lasing and selection have not yet been achieved. Free-space pumping is a stable and easy approach to coupling light into resonators and generating the lasing action in various narrow and harsh environments, such as a lowtemperature cryostat. However, until now, most of the reported WGM lasers that incorporate free-space pumping are multimodal due to the lack of mode-selection strategies.

Polymers are good hosts for various lasing gain dopants because of their advantages of easy processing, mechanical flexibility and low $\operatorname{cost}^{12,27-30}$. In this work, we use laser-interference excitation to develop a simple and general approach to realizing single-mode

${ }^{1}$ Shanghai Key Laboratory of Modern Optical Systems, Engineering Research Center of Optical Instrument and System (Ministry of Education), University of Shanghai for Science and Technology, Shanghai 200093, China; ${ }^{2}$ State Key Laboratory of Modern Optical Instrumentation, College of Optical Science and Engineering, Zhejiang University, Hangzhou 310027, China and ${ }^{3}$ State Key Laboratory of Precision Spectroscopy, East China Normal University, Shanghai 200062, China

Correspondence: F Gu, Email: fuxinggu@gmail.com; H Zeng, Email: hpzeng@phy.ecnu.edu.cn; S Zhuang, Email: slzhuang@yahoo.com

Received 14 December 2016; revised 27 March 2017; accepted 29 March 2017; accepted article preview online 31 March 2017 
WGM lasing in polymer bottle microresonators. When spatially overlapping the pump stripes with the intensity profiles of singlebottle WGMs, single-mode lasing can be efficiently generated and selected; attractive advantages of this system include a high side-mode suppression factor (SMSF), large tunability and low-lasing threshold.

\section{MATERIALS AND METHODS}

\section{Sample fabrication}

The gain material used is Rhodamine 6G (R6G)-doped epoxy resin with high viscosity and a refractive index $(n)$ of $\sim 1.53$ (Refs 9,13). Silica microfibers and fiber tapers are drawn from standard optical fibers (SMF-28, Corning, Hickory, NC, USA) using a flame-heating $\operatorname{method}^{27,31,32}$. The polymer bottle microresonators are fabricated using a self-assembly procedure, as shown in Figure 1a and $1 \mathrm{~b}$. First, a silica fiber taper is used to pick up a microdroplet of R6G-doped epoxy resin solution via micromanipulation ${ }^{27,31,32}$. When the microdroplet touches the suspended silica microfiber, the taper is drawn back, and a thin layer of the solution is left on the microfiber, and the solution shrinks to rapidly form a spindle-like shape due to surface tension (as shown in Supplementary Fig. S1). Finally, the droplet is solidified by heating at $60^{\circ} \mathrm{C}$.

Each shape of the as-fabricated bottle microresonators can be determined on the basis of three parameters: bottle outer diameter
$\left(D_{\text {out }}\right)$, microfiber diameter $\left(D_{\text {fiber }}\right)$ and neck-to-neck length $(L)$, as denoted in Figure 1c. Figure 1d shows a typical scanning electron microscope (SEM) image of a bottle microresonator with dimensions of $D_{\text {out }}=4.9 \mu \mathrm{m}, D_{\text {fiber }}=2.6 \mu \mathrm{m}$ and $L=9.2 \mu \mathrm{m}$, in which excellent surface smoothness is clearly seen. The shapes of the bottle microresonators can be tuned by adding or removing polymer solution using a fiber taper before heating solidification. Figure 1e shows that the $D_{\text {out }}$ of a bottle microresonator is changed from 3.2 to $9.5 \mu \mathrm{m}$. Thus, many bottle microresonators with different shapes can be fabricated along the silica microfiber. Figure if shows a picture of several bottle microresonators, with $D_{\text {out }}$ changed from $<2 \mu \mathrm{m}$ to nearly $10 \mu \mathrm{m}$. In this work, the bottle microresonators with $D_{\text {out }}$ ranging from 3 to $6 \mu \mathrm{m}$ are investigated.

\section{Laser-interference excitation}

The laser-interference excitation approach is illustrated in Figure $2 \mathrm{a}$. First, a $532-\mathrm{nm}$ pulsed laser $(5 \mathrm{~Hz}, 10 \mathrm{~ns})$ is divided into two collimated beams by a beam splitter. After being reflected by several reflection mirrors, the two counterpropagating beams are reflected by the corner of a knife-edge, right-angle prism (MRAK25-F01, Thorlabs, Newton, NJ, USA), which is actuated by a differential micrometer with $0.1 \mu \mathrm{m}$ sensitivity (DM-25L, Newport, Irvine, CA, USA), to form two parallel beams. Then, the two beams are focused onto a spot with a a

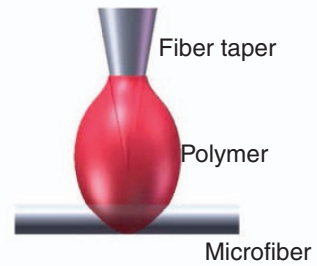

Shrink

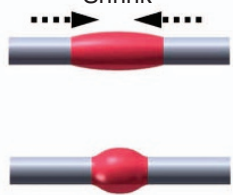

Bottle microresonator

C
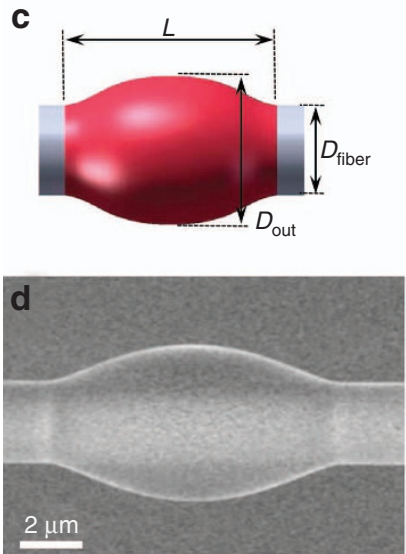
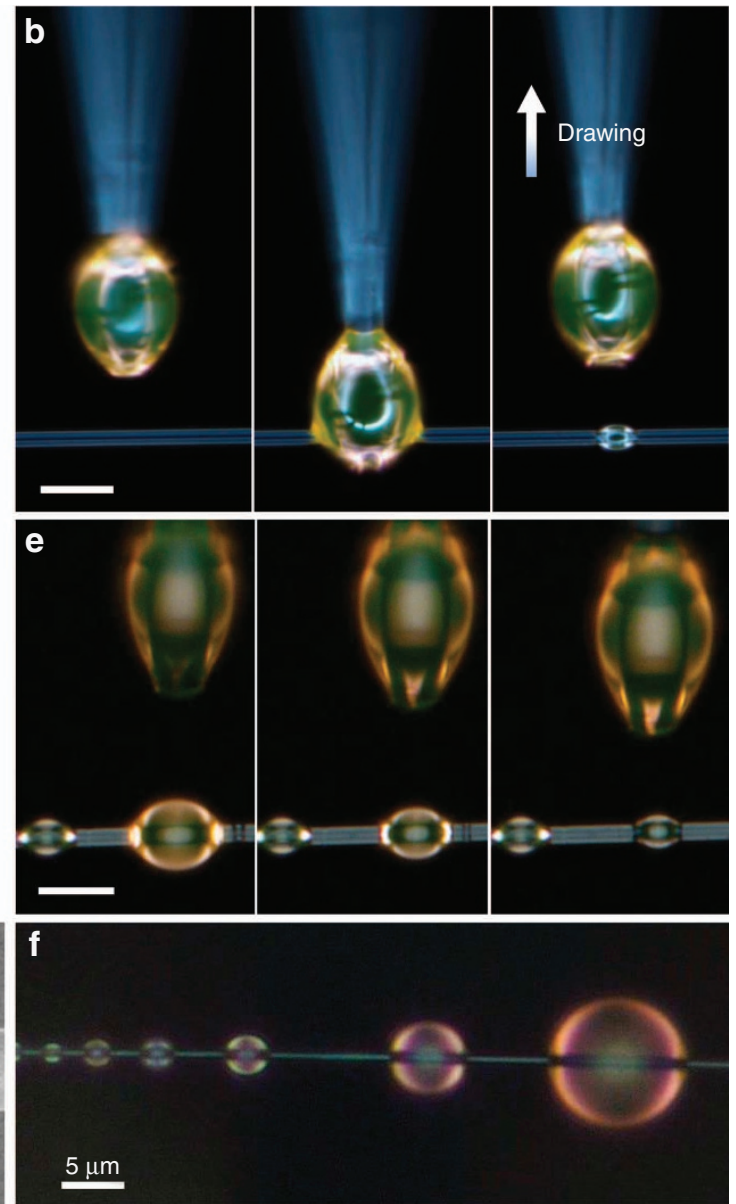

Figure 1 Fabrication and characterization of polymer bottle microresonators. (a) Schematic and (b) microscope images of fabricating polymer bottle microresonators using a self-assembly procedure. Scale bar $=10 \mu \mathrm{m}$. (c) Definitions of $L$, $D_{\text {out }}$ and $D_{\text {fiber }}$. (d) SEM image of a fabricated polymer bottle microresonator. (e) Shape tuning of bottle microresonators using a fiber taper before heating solidification. Scale bar $=10 \mu \mathrm{m}$. (f) $\mathrm{Microscope}$ image of polymer bottle microresonators with different $D_{\text {out }}$ values from $<2 \mu \mathrm{m}$ to nearly $10 \mu \mathrm{m}$. 
a

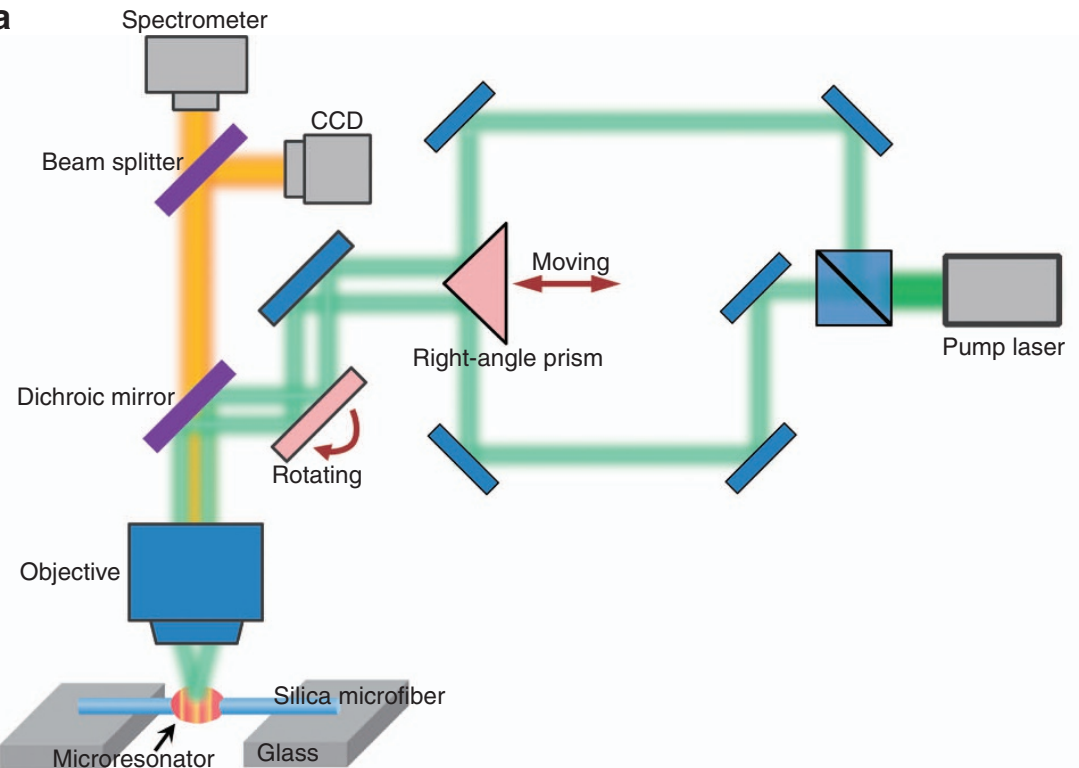

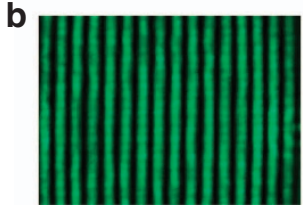

$0.85 \mu \mathrm{m}$

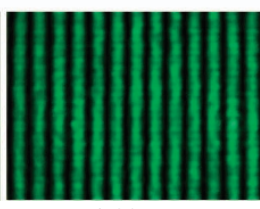

$1.32 \mu \mathrm{m}$

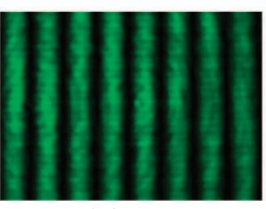

$2.15 \mu \mathrm{m}$

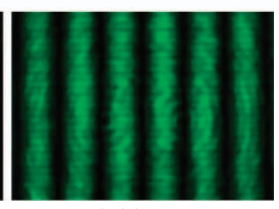

$2.89 \mu \mathrm{m}$

Figure 2 (a) Experimental setup for laser-interference excitation of polymer bottle microresonators. By moving the differential micrometer (shown in pink), $S_{\text {pump }}$ of the interference stripes can be adjusted. By rotating the reflection mirror (shown in pink), the position of the interference stripes along the long-axis direction of the bottle microresonator can be adjusted. (b) Microscope images of interference patterns obtained for four typical $S_{\text {pump }}$ values.

diameter of $\sim 30 \mu \mathrm{m}$ through a microscope objective $(\times 100, \mathrm{NA}=0.7)$ to produce interference patterns on the microresonator surface (the front focal plane of the objective). The bottle microresonators are suspended across a glass channel with a width of $\sim 500 \mu \mathrm{m}$. Their photoluminescence (PL) signals are collected using the same objectives and then delivered to a spectrometer (QE65 Pro, Ocean Optics, Winter Park, FL, USA, spectral resolution: $0.7 \mathrm{~nm}$ ) for spectral analysis and to a CCD camera for image capture ${ }^{31}$.

By moving the differential micrometers, the lateral displacement of the two collimated beams can be tuned, which finally determines the incident angle of the two beams at the focal plane and the spacing between interference patterns. Experimentally, the spacing $\left(S_{\text {pump }}\right)$ between the interference stripes can be precisely adjusted from $\sim 0.7$ to $3 \mu \mathrm{m}$. Figure $2 \mathrm{~b}$ shows typical microscope images of interference patterns for $S_{\text {pump }}$ values of $0.85,1.32,2.15$ and $2.89 \mu \mathrm{m}$, in which the intensity distribution along the long-axis direction follows sine functions with a visibility close to 1 , as is expected (Supplementary Fig. S2). By rotating a reflection mirror (shown in pink), the position of the interference patterns along the long-axis direction of bottle microresonators can also be precisely adjusted.

\section{RESULTS AND DISCUSSION}

Figure 3a shows the PL spectrum of the R6G-doped epoxy resin, indicating a broad gain spectrum with a full width at half maximum (FWHM) of $\sim 48 \mathrm{~nm}$. For a uniform pump that is commonly used, Figure $3 \mathrm{~b}$ shows typical emission spectra versus the peak-power density of pump light in a bottle microresonator with dimensions of $D_{\text {out }}=4.8 \mu \mathrm{m}, D_{\text {fiber }}=2.3 \mu \mathrm{m}$ and $L=7.5 \mu \mathrm{m}$. As the pump power increases above the threshold, a multimode-lasing action is observed.
The measured FWHM is $\sim 1.6 \mathrm{~nm}$ at the dominant peak wavelength $\left(\lambda_{\text {peak }}\right)$ of $595.9 \mathrm{~nm}$, which is limited by the spectral resolution of the spectrometer $(0.7 \mathrm{~nm})$. In Figure $3 \mathrm{c}$, the measured threshold at $\lambda_{\text {peak }}=595.9 \mathrm{~nm}$ is $0.09 \mathrm{MW} \mathrm{cm}^{-2}$ and is comparable to most values obtained for WGM microresonators with diameters larger than $10 \mu \mathrm{m}$ (Supplementary Fig. S3) ${ }^{9,13,33}$, suggesting that decreasing the dimensions of our bottle microresonators will not increase the lasing threshold, which is related to the low optical loss and high quality of bottle microresonators.

As illustrated in Figure $4 \mathrm{a}$, the prolate shape of a bottle microresonator supports highly non-degenerated WGMs (transverse modes) with spatially well-separated intensity along the longaxis direction ${ }^{16-18,24-26}$. Under the action of a uniform pump, the modes within the gain curve of resonators can be excited and a multimode-lasing behavior is observed (as demonstrated in Figure 3b). By using small-bottle microresonators with $D_{\text {out }}$ $<6 \mu \mathrm{m}$, the large FSR can keep only several transverse modes within the whole-cavity resonance range by pushing all other modes to the edge or out of the gain range, as shown in the right schematic of Figure 4a. Because different transverse modes have markedly different axial distributions, by engineering the pump intensity such that it produces an interference distribution on the microresonator surface (Figure $4 \mathrm{~b}$ ), the spacing between and axial positions of pump patterns can be spatially overlapped with the intensity profile of a desired WGM. Therefore, only the excited WGMs can lase, and other competing modes are suppressed. It is evident that such a bottle WGM laser is intrinsically single mode (right schematic of Figure 4b). 
To demonstrate this principle, the black line in Figure 5a (bottom) shows a representative lasing spectrum under the action of a uniform pump, measured in a bottle microresonator with dimensions of
$D_{\text {out }}=4.7 \mu \mathrm{m}, D_{\text {fiber }}=2.7 \mu \mathrm{m}$ and $L=7.5 \mu \mathrm{m}$, in which four strong lasing peaks are denoted. Its corresponding microscope image is shown in Figure 5b. Each bottle's WGMs can be defined by the axial a
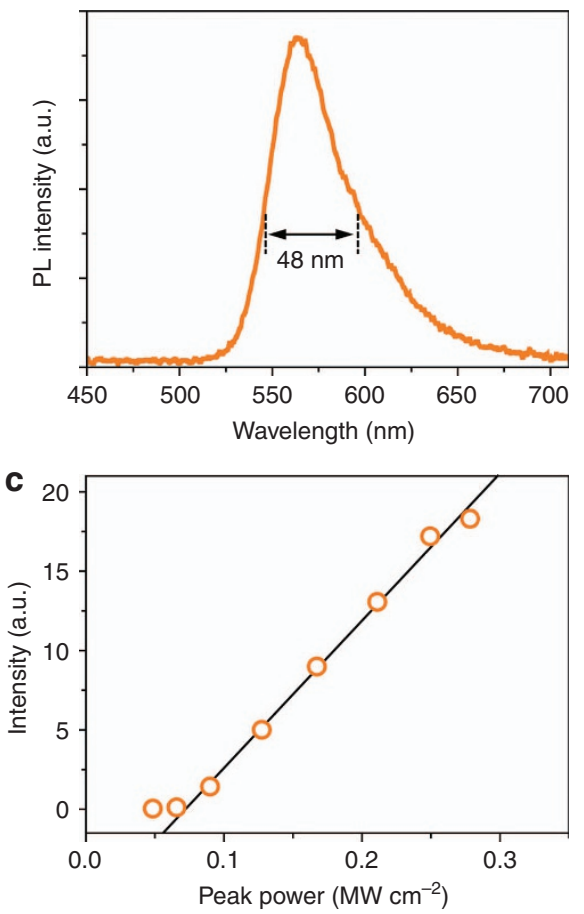

b

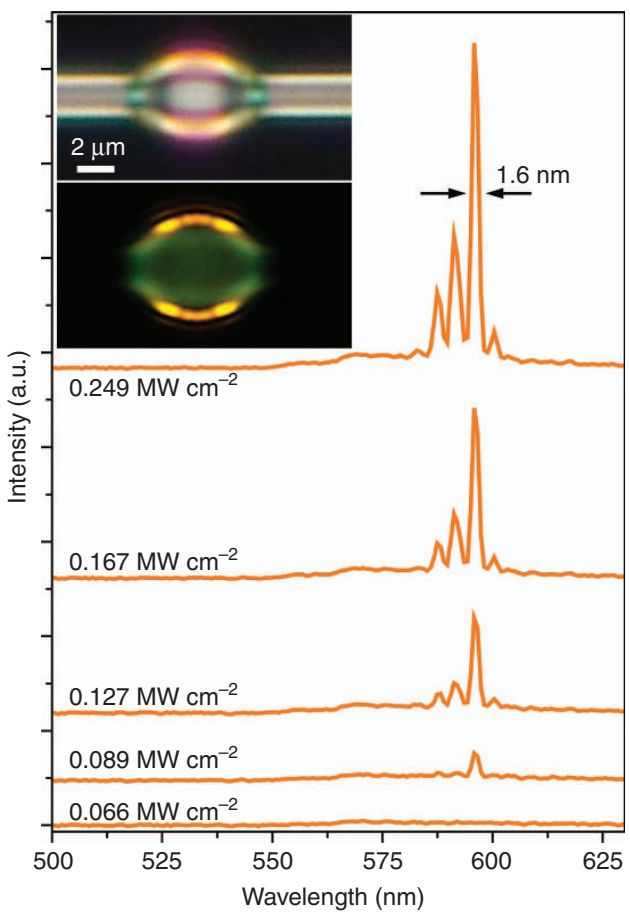

Figure 3 (a) PL spectrum of R6G-doped epoxy resin. (b) Emission spectra and microscope images of a polymer bottle microresonator $\left(D_{\text {out }}=4.8 \mu \mathrm{m}\right.$, $D_{\text {fiber }}=2.3 \mu \mathrm{m}$ and $L=7.5 \mu \mathrm{m}$ ) under uniform-pump excitation with different peak-power densities. Upper inset: bright-field microscope image of the polymer bottle microresonator; bottom inset: dark-field microscope image of its lasing generation. (c) Emission intensity at the 595.9-nm dominant peak versus the pump peak-power density.

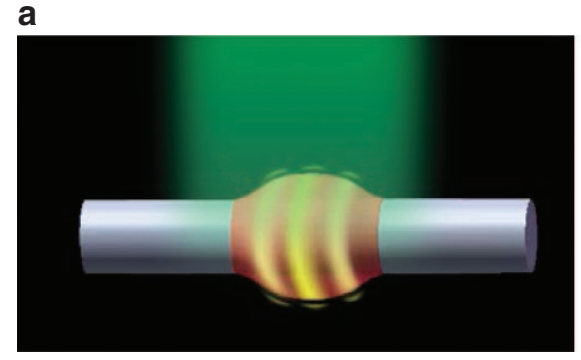

Uniform pump

b

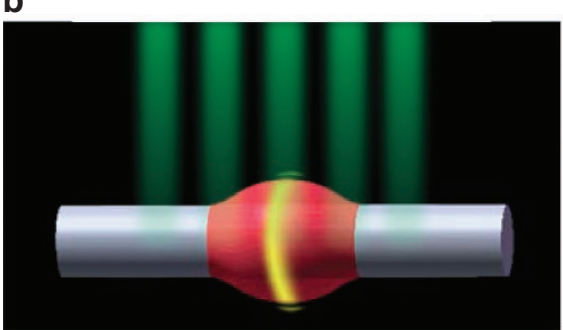

Engineered pump

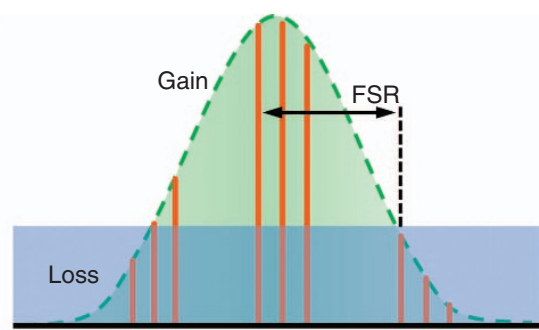

Multimode lasing

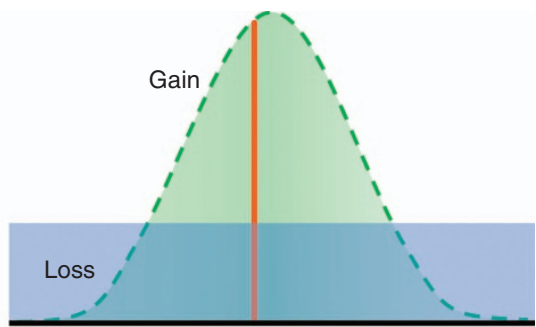

Single-mode lasing

Figure 4 Principle of single-mode WGM lasing in a polymer bottle microresonator. (a) Multimode-lasing behavior under the action of a uniform pump. Right schematic shows that by using small-bottle microresonators, the large FSR can keep only several transverse modes within the entire cavity resonance range by pushing all other modes to the edge or out of the gain range. (b) By engineering the pump intensity to modify the spatial gain profiles of bottle WGMs, the mode intensity profiles of single-bottle WGMs can be spatially overlapped with the pump stripes, intrinsically enabling single-mode lasing (right schematic). 
a

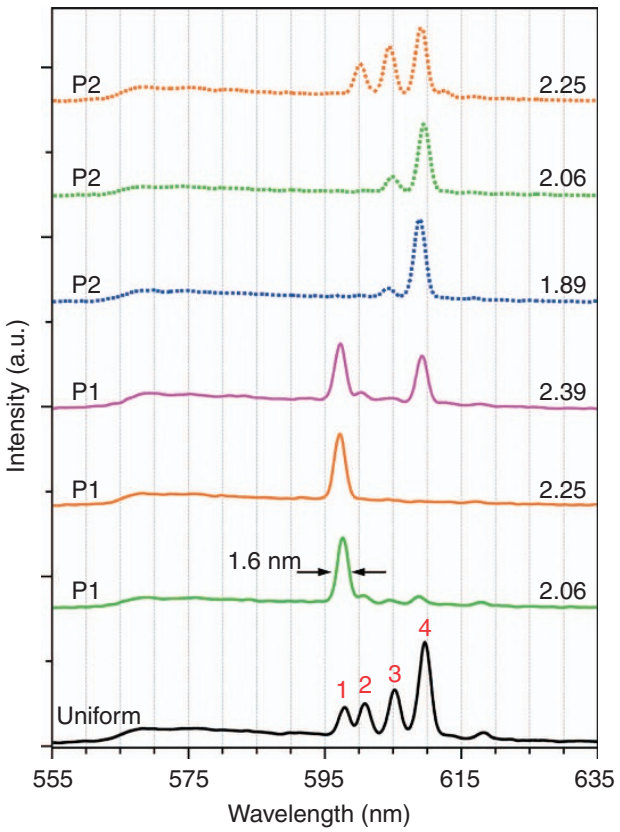

b

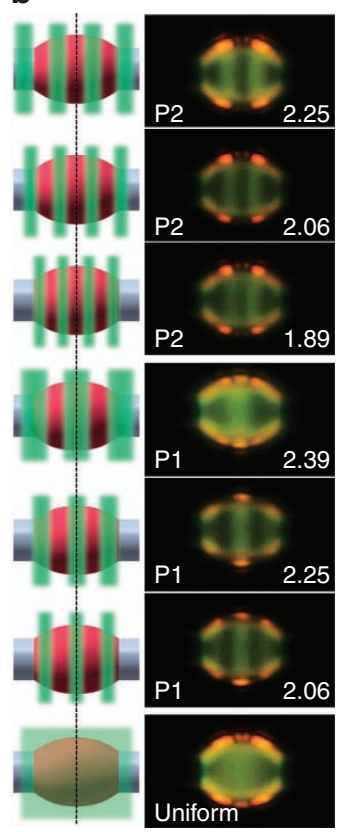

C
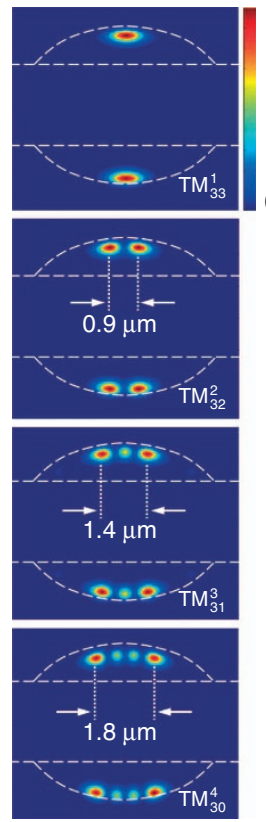

Figure 5 Observation of single-mode WGM lasing in a polymer bottle microresonator. (a) Lasing spectra and (b) their corresponding microscope images of a polymer bottle microresonator $\left(D_{\text {out }}=4.7 \mu \mathrm{m}, D_{\text {fiber }}=2.7 \mu \mathrm{m}\right.$ and $\left.L=7.5 \mu \mathrm{m}\right)$ under the action of a uniform and a laser-interference pump. The colored solid lines are recorded for different P1 patterns, and the colored dashed lines are recorded for different P2 patterns. The black dotted line in $\mathbf{b}$ denotes the centerline of the bottle microresonator. (c) Electric field-intensity distributions of $\mathrm{TM}_{33}^{1}, \mathrm{TM}_{32}^{2}, \mathrm{TM}_{31}^{3}$ and $\mathrm{TM}_{30}^{4}$ modes on the cross-plane of the bottle microresonator along its axis direction.

number $q$ and the azimuthal number $m$, which represent the number of intensity maxima along the axis and the half number of intensity maxima in the plane perpendicular to the axis, respectively ${ }^{18,24}$. By using a three-dimensional finite-difference time-domain (FDTD) method $^{34}$, we calculate the electromagnetic field distribution in the cavity. The simulations reveal that the peak of $1(597.9 \mathrm{~nm})$ corresponds well to the transverse-magnetic $\left(\mathrm{TM}_{m}^{q}\right)$ polarization mode $\mathrm{TM}_{33}^{1}$, and the peaks of $2(600.5 \mathrm{~nm}), 3(605.2 \mathrm{~nm})$ and $4(609.6 \mathrm{~nm})$ correspond well to $\mathrm{TM}_{32}^{2}, \mathrm{TM}_{31}^{3}$ and $\mathrm{TM}_{30}^{4}$, respectively (Supplementary Fig. S4). The FSR of the bottle microresonator is calculated as $17.2 \mathrm{~nm}$, which fits the function FSR $=\lambda_{\text {peak }}{ }^{2} / \pi n D_{\text {out }}$ well and confirms the WGM lasing mechanism. Figure $5 \mathrm{c}$ shows the electric field-intensity distributions of modes $1-4$ on the cross-plane of the bottle microresonator along its axis direction. The intensity of the $\mathrm{TM}_{33}^{1}$ mode is concentrated at the centerline, and the intensities of the $\mathrm{TM}_{32}^{2}, \mathrm{TM}_{31}^{3}$ and $\mathrm{TM}_{30}^{4}$ modes are distributed symmetrically at both sides of the centerline, with distances of $0.9,1.4$ and $1.8 \mu \mathrm{m}$ between the two maximum intensity positions, respectively.

For the case in which the spacing between and axial positions of interference patterns are changed, the lasing spectra and their corresponding microscope images are as shown in Figure $5 \mathrm{a}$ and $5 \mathrm{~b}$. For $S_{\text {pump }}=2.06 \mu \mathrm{m}$, when one stripe is located at the microresonator centerline (which can be simply called the P1 pattern), a pronounced single-mode lasing emission with $\lambda_{\text {peak }}=597.6 \mathrm{~nm}$ emerges. The SMSF is $\sim 9.3 \mathrm{~dB}$, and the FWHM is $\sim 1.6 \mathrm{~nm}$. The single bright spots at both the top and bottom edges of the bottle microresonator (Figure 5b) are attributed to the light scattering due to the imperfect surface quality and are expected to reveal the electromagnetic fieldintensity distribution inside the bottle microresonator (Figure 5c). Obviously, the scattering pattern matches the intensity distribution of the $\mathrm{TM}_{33}^{1}$ mode well. When $S_{\text {pump }}=2.25 \mu \mathrm{m}$, a single-mode emission with $\lambda_{\text {peak }}=597.4 \mathrm{~nm}$ and a SMSF of $16.2 \mathrm{~dB}$ are achieved, which is also due to the $\mathrm{TM}_{33}^{1}$ mode. Another transverse mode can be selected when two stripes are symmetrically located on both sides of the centerline (which can be simply called the P2 patterns). For $S_{\text {pump }}=1.89 \mu \mathrm{m}$, an obvious single-mode lasing emission emerges, with $\lambda_{\text {peak }}=608.9 \mathrm{~nm}$ and a SMSF of $9.9 \mathrm{~dB}$. The four bright light spots at both the top and bottom edges share the same signature as the intensity distribution of the $\mathrm{TM}_{30}^{4}$ mode. When $S_{\text {pump }}=2.06 \mu \mathrm{m}$, a single-mode emission with $\lambda_{\text {peak }}=609.3 \mathrm{~nm}$ and a SMSF of $6.7 \mathrm{~dB}$ is observed, which also arises from the $\mathrm{TM}_{30}^{4}$ mode.

If we further increase the spacing, the widths of the stripes become so large that the discrepancy of overlapping with different modes vanishes, which induces multimode lasing. As shown in Figure 5a and $5 \mathrm{~b}$, when using the P1 pattern with $S_{\text {pump }}=2.39 \mu \mathrm{m}$, two strong peaks corresponding to the $\mathrm{TM}_{33}^{1}$ and $\mathrm{TM}_{30}^{4}$ modes are observed for P1 patterns, whereas when using the P2 pattern with $S_{\text {pump }}=2.25 \mu \mathrm{m}$, three strong $\mathrm{TM}_{32}^{2}, \mathrm{TM}_{31}^{3}$ and $\mathrm{TM}_{30}^{4}$ transverse modes are observed. Similarly, when using a small spacing, such as $S_{\text {pump }}=1.77 \mu \mathrm{m}$, the dense stripes induce multimode lasing (Supplementary Fig. S5).

By carefully tuning the spacing between and axial positions of pump patterns, a single-mode lasing with a SMSF as high as $21.7 \mathrm{~dB}$ is obtained from a bottle microresonator with dimensions of $D_{\text {out }}=4.5 \mu \mathrm{m}, D_{\text {fiber }}=1.6 \mu \mathrm{m}$ and $L=5.1 \mu \mathrm{m}$ (Figure 6a). The selection of single modes can be further improved by using ultranarrowlinewidth pump lasers or by using optimized pump patterns that have been used in single-mode random lasers ${ }^{35-37}$. The thresholds of the selected single-mode lasing are also investigated. As shown in Figure 6b, the measured threshold of a bottle microresonator $\left(D_{\text {out }}=4.4 \mu \mathrm{m}, D_{\text {fiber }}=2.1 \mu \mathrm{m}\right.$ and $\left.L=5.4 \mu \mathrm{m}\right)$ is $\sim 1.62 \mathrm{MW} \mathrm{cm}^{-2}$ under the action of a uniform pump and reduces to $\sim 1.07 \mathrm{MW} \mathrm{cm}^{-2}$ under the action of a P1-pattern pump, which occurs due to the high 

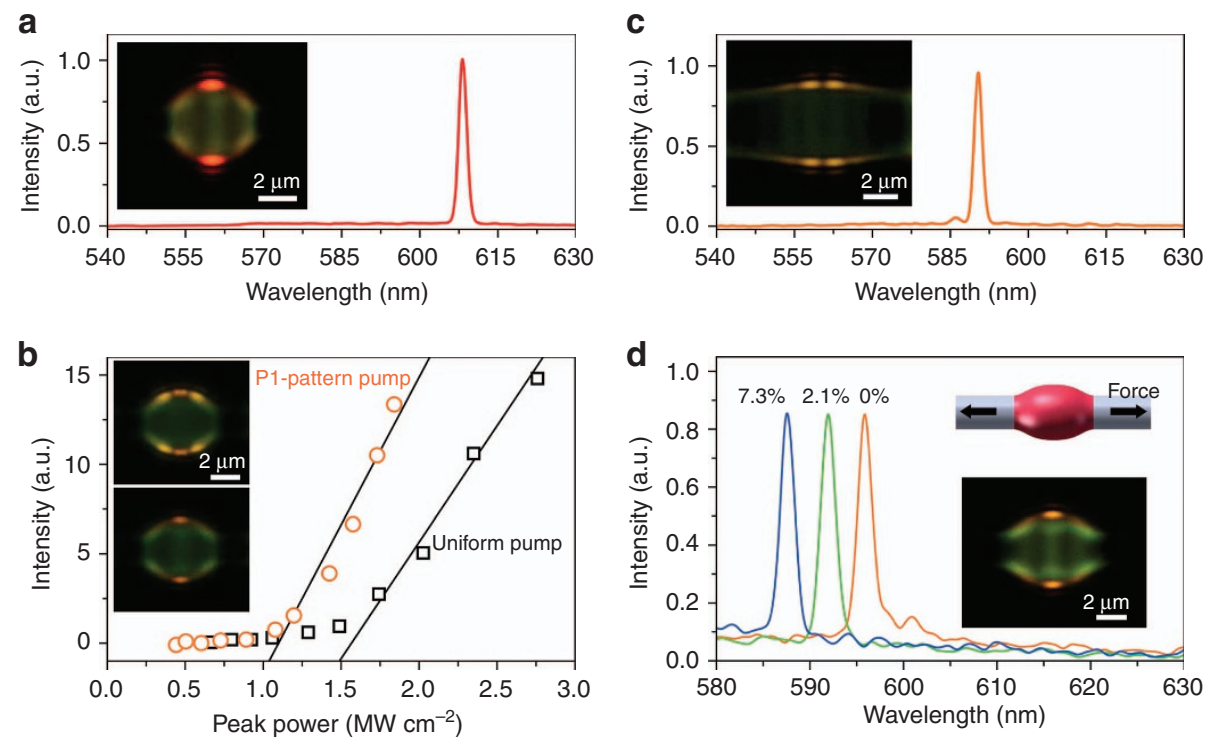

Figure 6 (a) Single-mode lasing with a SMSF as high as $21.7 \mathrm{~dB}$, obtained from a bottle microresonator $\left(D_{\text {out }}=4.5 \mu \mathrm{m}, D_{\text {fiber }}=1.6 \mu \mathrm{m}\right.$ and $\left.L=5.1 \mu \mathrm{m}\right)$. Inset shows its lasing microscope image. (b) Lasing threshold comparison between the uniform and the P1-pattern pump conditions. Insets show the lasing microscope images for (upper) the uniform and (bottom) the P1-pattern pump. (c) Higher-order single transverse-mode lasing from a more prolate bottle microresonator $\left(D_{\text {out }}=4.6 \mu \mathrm{m}, D_{\text {fiber }}=3.7 \mu \mathrm{m}\right.$ and $\left.L=10.4 \mu \mathrm{m}\right)$. Inset shows its lasing microscope image. (d) Spectral shift of a lasing peak from 595.8 to $587.4 \mathrm{~nm}$ for a tensile strain increase from 0 to $\sim 7.3 \%$. Upper inset shows a schematic of strain sensing; bottom inset shows its lasing microscope image.

overlap of the pump stripes and the WGM intensity profiles that make the pump energy usage more efficient.

This laser-interference excitation approach is very general and reproducible and, in principle, valid for almost arbitrary small-bottle microresonators (here, $D_{\text {out }}$ is $<6 \mu \mathrm{m}$ ). For example, Figure $6 \mathrm{c}$ shows a higher-order single transverse-mode lasing from a more prolate bottle microresonator with dimensions of $D_{\text {out }}=4.6 \mu \mathrm{m}$, $D_{\text {fiber }}=3.7 \mu \mathrm{m}$ and $L=10.4 \mu \mathrm{m}$, for which the SMSF is measured to be $14.1 \mathrm{~dB}$ at $\lambda_{\text {peak }}=590.5 \mathrm{~nm}$. In addition, the microfiber that supports the bottle microresonators is not limited to a silica material. For example, Figure $6 \mathrm{~d}$ shows single-mode lasing with $\lambda_{\text {peak }}=595.8 \mathrm{~nm}$ from a bottle microresonator $\left(D_{\text {out }}=4.9 \mu \mathrm{m}\right)$ with a $2.6-\mu \mathrm{m}$-diameter poly(vinyl chloride) (PVC) microfiber ${ }^{32}$. Benefitting from the high-mechanical pliability, the lasing emission can be tuned by pulling the PVC microfiber along its length direction (Supplementary Fig. S6) ${ }^{38}$. For a tensile strain increase from 0 to $\sim 7.3 \%$, the 595.8 -nm lasing peak shifts to $587.4 \mathrm{~nm}$ while maintaining its FWHM, which corresponds to a change of over $50 \%$ of the FSR $(\sim 15.3 \mathrm{~nm})$ and suggests a feasible approach to broad, tunable singlemode lasers.

\section{CONCLUSIONS}

In conclusion, we have demonstrated intrinsic single-mode WGM lasing in polymer bottle microresonators by engineering the pump intensity to modify the spatial gain profiles of bottle WGMs. When their mode intensity profiles are spatially overlapped with the pump stripes, single-bottle WGMs can be efficiently selected to lase, with attractive advantages including a high SMSF (over $20 \mathrm{~dB}$ ), large tunability (over $8 \mathrm{~nm}$ ) and low-lasing threshold. Only two parameters, that is, the spacing between and positions of interference stripes, need to be adjusted. In addition, this approach is precise and reversibly controllable and does not need a complex facility design and expensive components. Moreover, the mechanism can, in principle, be adopted for other types of bottle microresonators, such as hollow-bubble or tube resonators ${ }^{20,23-25}$, and for other gain media, such as graphene quantum $\operatorname{dots}^{39}$, up-conversion nanocrystals ${ }^{40}$ and perovskite nanoparticles ${ }^{41}$ ranging from their solid form to their liquid form ${ }^{42}$. Therefore, our simple and general approach may have a variety of promising applications, ranging from tunable single-mode lasing and sensing to nonlinear optics ${ }^{43}$.

\section{CONFLICT OF INTEREST}

The authors declare no conflict of interest.

\section{ACKNOWLEDGEMENTS}

This work is supported by the National Natural Science Foundation of China (11674230), 973 Program (2015CB352001) and National Natural Science Foundation of China (11434005).

1 Armani DK, Kippenberg TJ, Spillane SM, Vahala KJ. Ultra-high-Q toroid microcavity on a chip. Nature 2003; 421: 925-928.

2 He LN, Öezdemir ŞK, Yang L. Whispering gallery microcavity lasers. Laser Photonics Rev 2013; 7: 60-82.

3 Yang SC, Wang Y, Sun HD. Advances and prospects for whispering gallery mode microcavities. Adv Opt Mater 2015; 3: 1136-1162.

4 Jiang XF, Zou CL, Wang L, Gong QH, Xiao YF. Whispering-gallery microcavities with unidirectional laser emission. Laser Photonics Rev 2016; 10: 40-61.

5 Grossmann T, Schleede S, Hauser M, Christiansen MB, Vannahme C et al. Lowthreshold conical microcavity dye lasers. Appl Phys Lett 2010; 97: 063304.

6 Bog U, Laue T, Grossmann T, Beck T, Wienhold T et al. On-chip microlasers for biomolecular detection via highly localized deposition of a multifunctional phospholipid ink. Lab Chip 2013; 13: 2701-2707.

7 Li B-B, Clements WR, Yu X-C, Shi K, Gong Q et al. Single nanoparticle detection using split-mode microcavity Raman lasers. Proc Natl Acad Sci USA 2014; 111: 14657-14662.

8 Chang L, Jiang XS, Hua SY, Yang C, Wen JM et al. Parity-time symmetry and variable optical isolation in active-passive-coupled microresonators. Nat Photonics 2014; 8 : 524-529.

9 Ta VD, Chen R, Sun HD. Tuning whispering gallery mode lasing from self-assembled polymer droplets. Sci Rep 2013; 3: 1362.

10 Shang L, Liu LY, Xu L. Single-frequency coupled asymmetric microcavity laser. Opt Lett 2008; 33: 1150-1152. 
$11 \mathrm{Li} \mathrm{H}$, Shang L, Tu X, Liu LY, Xu L. Coupling variation induced ultrasensitive label-free biosensing by using single mode coupled microcavity laser. J Am Chem Soc 2009; 131: 16612-16613.

12 Grossmann T, Wienhold T, Bog U, Beck T, Friedmann C et al. Polymeric photonic molecule super-mode lasers on silicon. Light Sci App/ 2013; 2: e82.

13 Ta VD, Chen R, Sun HD. Coupled polymer microfiber lasers for single mode operation and enhanced refractive index sensing. Adv Opt Mater 2014; 2: 220-225.

14 Feng L, Wong ZJ, Ma R-M, Wang Y, Zhang X. Single-mode laser by parity-time symmetry breaking. Science 2014; 346: 972-975.

15 Hodaei H, Miri M-A, Heinrich M, Christodoulides DN, Khajavikhan M. Parity-timesymmetric microring lasers. Science 2014; 346: 975-978.

16 Sumetsky M. Whispering-gallery-bottle microcavities: the three-dimensional etalon. Opt Lett 2004; 29: 8-10.

17 Strelow C, Rehberg H, Schultz CM, Welsch H, Heyn C et al. Optical microcavities formed by semiconductor microtubes using a bottlelike geometry. Phys Rev Lett 2008; 101: 127403.

18 Pöellinger M, O'Shea D, Warken F, Rauschenbeutel A. Ultrahigh- $Q$ tunable whisperinggallery-mode microresonator. Phys Rev Lett 2009; 103: 053901.

19 Sumetsky M. Delay of light in an optical bottle resonator with nanoscale radius variation: dispersionless, broadband, and low loss. Phys Rev Lett 2013; 111: 163901.

20 Kim KH, Bahl G, Lee W, Liu J, Tomes M et al. Cavity optomechanics on a microfluidic resonator with water and viscous liquids. Light Sci Appl 2013; 2: e110.

21 Wang Y, Zhang K, Zhou S, Wu Y-H, Chi M-B et al. Coupled-mode induced transparency in a bottle whispering-gallery-mode resonator. Opt Lett 2016; 41: 1825-1828.

22 Riesen N, Zhang WQ, Monro TM. Dispersion in silica microbubble resonators. Opt Lett 2016; 41: 1257-1260.

23 Yang Y, Ooka Y, Thompson RM, Ward JM, Chormaic SN. Degenerate four-wave mixing in a silica hollow bottle-like microresonator. Opt Lett 2016; 41: 575-578.

24 Murugan GS, Wilkinson JS, Zervas MN. Selective excitation of whispering gallery modes in a novel bottle microresonator. Opt Express 2009; 17: 11916-11925.

25 Murugan GS, Petrovich MN, Jung Y, Wilkinson JS, Zervas MN. Hollow-bottle optical microresonators. Opt Express 2011; 19: 20773-20784.

26 Ding M, Murugan GS, Brambilla G, Zervas MN. Whispering gallery mode selection in optical bottle microresonators. Appl Phys Lett 2012; 100: 081108.

27 Gu FX, Yu HK, Wang P, Yang ZY, Tong LM. Light-emitting polymer single nanofibers via waveguiding excitation. ACS Nano 2010; 4: 5332-5338.

28 Yan YL, Zhao YS. Organic nanophotonics: from controllable assembly of functional molecules to low-dimensional materials with desired photonic properties. Chem Soc Rev 2014; 43: 4325-4340.

29 Garreau A, Duvail J-L. Recent advances in optically active polymer-based nanowires and nanotubes. Adv Opt Mater 2014; 2: 1122-1140.
30 Persano L, Camposeo A, Pisignano D. Active polymer nanofibers for photonics, electronics, energy generation and micromechanics. Prog Polym Sci 2015; 43: 48-95.

$31 \mathrm{Gu}$ FX, Zhang L, Zhu YB, Zeng HP. Free-space coupling of nanoantennas and whispering-gallery microcavities with narrowed linewidth and enhanced sensitivity. Laser Photonics Rev 2015; 9: 682-688.

32 Gu FX, Zhang L, Yin XF, Tong LM. Polymer single-nanowire optical sensors. Nano Lett 2008; 8: 2757-2761.

33 Ta VD, Chen R, Ma L, Ying YJ, Sun HD. Whispering gallery mode microlasers and refractive index sensing based on single polymer fiber. Laser Photonics Rev 2013; 7: 133-139.

34 Lin X, Fang W. Localized high-Q modes in conical microcavities. Opt Commun 2016; 381: 169-173.

35 Bachelard N, Gigan S, Noblin X, Sebbah P. Adaptive pumping for spectral control of random lasers. Nat Phys 2014; 10: 426-431.

36 Hisch T, Liertzer M, Pogany D, Mintert F, Rotter S. Pump-controlled directional light emission from random lasers. Phys Rev Lett 2013; 111: 023902.

37 Liew SF, Redding B, Ge L, Solomon GS, Cao H. Active control of emission directionality of semiconductor microdisk lasers. Appl Phys Lett 2014; 104: 231108.

38 Gu FX, Yu HK, Fang W, Tong LM. Nanoimprinted polymer micro/nanofiber Bragg gratings for high-sensitivity strain sensing. IEEE Photonics Technol Lett 2013; 25: 22-24.

39 Shen JH, Zhu YH, Yang XL, Li CZ. Graphene quantum dots: emergent nanolights for bioimaging, sensors, catalysis and photovoltaic devices. Chem Commun 2012; 48: 3686-3699.

40 Zhou B, Shi BY, Jin DY, Liu XG. Controlling upconversion nanocrystals for emerging applications. Nat Nanotechnol 2015; 10: 924-936.

41 Sutherland BR, Sargent EH. Perovskite photonic sources. Nat Photonics 2016; 10 : 295-302.

42 Fan XD, Yun SH. The potential of optofluidic biolasers. Nat Methods 2014; 11: 141-147.

43 Torres-Company V, Weiner AM. Optical frequency comb technology for ultra-broadband radio-frequency photonics. Laser Photonics Rev 2014; 8: 368-393.

(c) (i) (2) This work is licensed under a Creative Commons AttributionBY NC SA NonCommercial-ShareAlike 4.0 International License. The images or other third party material in this article are included in the article's Creative Commons license, unless indicated otherwise in the credit line; if the material is not included under the Creative Commons license, users will need to obtain permission from the license holder to reproduce the material. To view a copy of this license, visit http:// creativecommons.org/licenses/by-nc-sa/4.0/

C) The Author(s) 2017

Supplementary Information for this article can be found on the Light: Science \& Applications' website (http://www.nature.com/lsa). 